Bull. Korean Math. Soc. 52 (2015), No. 4, pp. 1123-1132

http://dx.doi.org/10.4134/BKMS.2015.52.4.1123

\title{
DERIVATIONS ON CONVOLUTION ALGEBRAS
}

\author{
Mohammad Javad Mehdipour and Zahra Saeedi
}

ABSTRACT. In this paper, we investigate derivations on the noncommutative Banach algebra $L_{0}^{\infty}(\omega)^{*}$ equipped with an Arens product. As a main result, we prove the Singer-Wermer conjecture for the noncommutative Banach algebra $L_{0}^{\infty}(\omega)^{*}$. We then show that a derivation on $L_{0}^{\infty}(\omega)^{*}$ is continuous if and only if its restriction to $\operatorname{rad}\left(L_{0}^{\infty}(\omega)^{*}\right)$ is continuous. We also prove that there is no nonzero centralizing derivation on $L_{0}^{\infty}(\omega)^{*}$. Finally, we prove that the space of all inner derivations of $L_{0}^{\infty}(\omega)^{*}$ is continuously homomorphic to the space $L_{0}^{\infty}(\omega)^{*} / L^{1}(\omega)$.

\section{Introduction}

Let $\omega$ be a weight function on $\mathbb{R}^{+}=[0, \infty)$, i.e., a continuous function $\omega: \mathbb{R}^{+} \rightarrow[1, \infty)$ so that $\omega(0)=1$ and

$$
w(x+y) \leq w(x) w(y)
$$

for all $x, y \in \mathbb{R}^{+}$. Let also $L^{1}(\omega)$ be the Banach space of all Lebesgue measurable functions $\phi$ on $\mathbb{R}^{+}$such that

$$
\|\phi\|_{1, \omega}=\int_{0}^{\infty}|\phi(x)| w(x) d x<\infty .
$$

Then $L^{1}(\omega)$ with the norm $\|\cdot\|_{1, \omega}$ and the convolution product "*" defined by

$$
\phi * \psi(x)=\int_{0}^{x} \phi(y) \psi(x-y) d y \quad\left(x \in \mathbb{R}^{+}\right)
$$

is a Banach algebra. We also assume that $L_{0}^{\infty}(\omega)$ is the Banach space of all Lebesgue measurable functions $f$ on $\mathbb{R}^{+}$such that

$$
\left\|f \chi_{(x, \infty)}\right\|_{\infty, \omega}=\operatorname{ess} \sup \left\{f(y) \chi_{(x, \infty)}(y) / \omega(y): y \in \mathbb{R}^{+}\right\} \rightarrow 0
$$

as $x \rightarrow \infty$. For every $f \in L_{0}^{\infty}(\omega)$ and $\phi \in L^{1}(\omega)$, the function $f \circ \phi$ defined by

$$
f \circ \phi(x)=\int_{0}^{\infty} f(x+y) \phi(y) d y \quad\left(x \in \mathbb{R}^{+}\right)
$$

Received April 8, 2014; Revised January 16, 2015

2010 Mathematics Subject Classification. Primary 47B47, 46H40, 16W25.

Key words and phrases. derivation, inner derivation, centralizing, automatic continuity. 
is an element in $C_{0}(\omega)$, the Banach space of all continuous complex-valued functions $f$ on $\mathbb{R}^{+}$such that $f / \omega$ vanishes at infinity; see [10]. So, we may endow the dual of $L_{0}^{\infty}(\omega)$, represented by $L_{0}^{\infty}(\omega)^{*}$, with the first Arens product "." defined as follows. For any $\phi$ in $L^{1}(\omega), f$ in $L_{0}^{\infty}(\omega)$ and $m, n$ in $L_{0}^{\infty}(\omega)^{*}$, the element $m \cdot n$ is defined by

$$
\langle m \cdot n, f\rangle=\langle m, n f\rangle,
$$

where $\langle n f, \phi\rangle=\langle n, f \circ \phi\rangle$. Then $L_{0}^{\infty}(\omega)^{*}$ with this product is a Banach algebra; see [10]; see also Lau and Pym [8] for the locally compact group case. Note that since $\phi \cdot \psi=\phi * \psi$ for $\phi, \psi \in L^{1}(\omega), L^{1}(\omega)$ may be regarded as a subspace of $L_{0}^{\infty}(\omega)^{*}$ and then $L^{1}(\omega)$ is a closed ideal in $L_{0}^{\infty}(\omega)^{*}[10]$.

Since $\omega(x) \geq 1$ for all $x \in \mathbb{R}^{+}$, the Banach algebra $L^{1}(\omega)$ has a bounded approximate identity, for example the sequence $\left\{i \chi_{(0,1 / i)}\right\}_{i \in \mathbb{N}}$ is one of them; see [14]. We denote by $\Lambda\left(L_{0}^{\infty}(\omega)^{*}\right)$ the set of all weak* ${ }^{*}$ cluster points of an approximate identity in $L^{1}(\omega)$ bounded by one. Note that for any $n \in L_{0}^{\infty}(\omega)^{*}$ and $\phi \in L^{1}(\omega)$, the maps

$$
m \mapsto m \cdot n \quad \text { and } \quad m \mapsto \phi \cdot m
$$

are weak*-weak* continuous on $L_{0}^{\infty}(\omega)^{*}$. Hence if $u \in \Lambda\left(L_{0}^{\infty}(\omega)^{*}\right)$, then $u$ is a mixed identity with norm one, that is, $\|u\|=1$ and $u \cdot \phi=\phi \cdot u=\phi$ for all $\phi \in L^{1}(\omega)$. Goldstine's theorem implies that every mixed identity for $L_{0}^{\infty}(\omega)^{*}$ is a right identity for $L_{0}^{\infty}(\omega)^{*}$. Also, note that if $u$ is a right identity for $L_{0}^{\infty}(\omega)^{*}$ and $\left(e_{\alpha}\right)$ is a bounded net in $L^{1}(\omega)$ such that $e_{\alpha} \rightarrow u$ in the weak ${ }^{*}$ topology of $L_{0}^{\infty}(\omega)^{*}$, then for every $\phi \in L^{1}(\omega)$, we have

$$
\phi \cdot e_{\alpha} \rightarrow \phi
$$

in the weak topology. Passing to convex combinations, we can suppose that $\phi \cdot e_{\alpha} \rightarrow \phi$ in the norm topology. This together with the fact that $L^{1}(\omega)$ is a commutative Banach algebra shows that $\left(e_{\alpha}\right)$ is a bounded approximate identity for $L^{1}(\omega)$. Thus $u \in \Lambda\left(L_{0}^{\infty}(\omega)^{*}\right)$. These facts can be summarized by saying that $u \in \Lambda\left(L_{0}^{\infty}(\omega)^{*}\right)$ if and only if it is a right identity for $L_{0}^{\infty}(\omega)^{*}$ with norm one or, equivalently, $u$ is a mixed identity with norm one.

By $M(\omega)$ we mean the Banach space of all complex regular Borel measures $\mu$ on $\mathbb{R}^{+}$such that

$$
\|\mu\|_{\omega}=\int_{0}^{\infty} \omega(x) d|\mu|(x)<\infty,
$$

where $|\mu|$ denotes the total variation of $\mu$. By the usual way, the Banach space $M(\omega)$ can be identified with the dual of $C_{0}(\omega)$. So, we may define the convolution multiplication "*" by

$$
\langle\mu * \nu, g\rangle=\int_{0}^{\infty} \int_{0}^{\infty} g(x+y) d \mu(x) d \nu(y)
$$

for all $\mu, \nu \in M(\omega)$ and $g \in C_{0}(\omega)$. Then the Banach space $M(\omega)$ with the convolution product $*$ and the norm $\|\cdot\|_{\omega}$ is a commutative Banach algebra. Let us remark from [11, Theorem 3.6(i) and Example 4.13(c)] that if $u$ is an 
element of $\Lambda\left(L_{0}^{\infty}(\omega)^{*}\right)$, then $u \cdot L_{0}^{\infty}(\omega)^{*}$ is isometrically isomorphic to $M(\omega)$. This implies that $u \cdot L_{0}^{\infty}(\omega)^{*}$ is commutative and so for every $k, m, n \in L_{0}^{\infty}(\omega)^{*}$

$$
k \cdot m \cdot n=k \cdot u \cdot m \cdot u \cdot n=k \cdot u \cdot n \cdot u \cdot m=k \cdot n \cdot m .
$$

Let $\operatorname{rAnn}\left(L_{0}^{\infty}(\omega)^{*}\right)$ be the set of all $r \in L_{0}^{\infty}(\omega)^{*}$ such that $L_{0}^{\infty}(\omega)^{*} \cdot r=\{0\}$. For every $m \in L_{0}^{\infty}(\omega)^{*}, f \in L_{0}^{\infty}(\omega)$ and $\phi \in L^{1}(\omega)$, we have

$$
\langle\phi \cdot m, f\rangle=\langle\phi, m f\rangle=\langle m f, \phi\rangle=\langle m, f \circ \phi\rangle .
$$

Also it is well-known from [11, Proposition 2.3(b) and Example 4.13(c)] that

$$
L_{0}^{\infty}(\omega) \circ L^{1}(\omega)=C_{0}(\omega) .
$$

These facts show that

$$
\operatorname{rAnn}\left(L_{0}^{\infty}(\omega)^{*}\right)=C_{0}(\omega)^{\perp}:=\left\{r \in L_{0}^{\infty}(\omega)^{*}:\left.r\right|_{C_{0}(\omega)}=0\right\} .
$$

Let us recall from [11, Theorem 3.6(iv) and Example 4.13(c)] that if $u \in$ $\Lambda\left(L_{0}^{\infty}(\omega)^{*}\right)$, then the Banach space $L_{0}^{\infty}(\omega)^{*}$ is the Banach space direct sum of $u \cdot L_{0}^{\infty}(\omega)^{*}$ and $\operatorname{rAnn}\left(L_{0}^{\infty}(\omega)^{*}\right)$. if

Let $A$ be a Banach algebra; a linear mapping $D: A \rightarrow A$ is called a derivation

$$
D(a b)=D(a) b+a D(b) .
$$

In 1955, Singer and Wermer [17] proved that every continuous derivation on a commutative Banach algebra maps the algebra into its radical. They conjectured that the continuity requirement for the derivations is unnecessary and can be removed. Johnson [5] showed the automatic continuity of derivations of commutative semisimple Banach algebras and so the conjecture can be established for these types of Banach algebras. Finally in 1988, Thomas [19] gave an affirmative answer to the Singer-Wermer conjecture. Obviously, because of inner derivations, this result does not remain valid for noncommutative Banach algebras. There are, however, various noncommutative versions of the SingerWermer theorem for Banach algebras [11, 12, 16]. For example, Sinclair [16] proved that every continuous derivation of a Banach algebra $A$ leaves primitive ideals of $A$ invariant. Mathieu and Runde [12] gave another noncommutative extension of the Singer-Wermer theorem: Every centralizing derivation on a noncommutative Banach algebra has its image in the radical of algebra; see Posner [15] for the noncommutative version of the Singer-Wermer theorem for prime rings; see also $[1,6,7,20]$ for range inclusion results for derivations on not necessarily commutative Banach algebras.

Isik and et al. [4] gave some interesting results on the structure of the Banach algebra $L^{\infty}(G)^{*}$, for an infinite compact group $G$. Lau and Pym [8] introduced the subspace $L_{0}^{\infty}(G)$ of $L^{\infty}(G)$ consisting of bounded measurable functions on locally compact group $G$ that vanish at infinity. For a locally compact group, they proved most of the results obtained in [4] for $L_{0}^{\infty}(G)^{*}$. In fact, they introduced a sensible replacement for $L^{\infty}(G)$, when $G$ is compact. Maghsoudi and et al. [10] have introduced and studied a semigroup analogue of $L_{0}^{\infty}(G)^{*}$. They showed that some aspects of the theory of $L^{\infty}(G)^{*}$ when $G$ is 
a compact group hold for $L_{0}^{\infty}(\omega)^{*}$. Other aspects of analysis on these Banach algebras have been studied by many authors; see for example, [9, 13, 18].

Since $\operatorname{rAnn}\left(L_{0}^{\infty}(\omega)^{*}\right)=C_{0}(\omega)^{\perp}$, an easy application of the Hahn-Banach's theorem shows that there is a non-zero element $r \in \operatorname{rAnn}\left(L_{0}^{\infty}(\omega)^{*}\right)$; see for example [2, Corollary 6.8 of Chapter 3]. Hence for every $u \in \Lambda\left(L_{0}^{\infty}(\omega)^{*}\right)$, we have $u \cdot r=0, r \cdot u=r$ and

$$
r \cdot L_{0}^{\infty}(\omega)^{*} \cdot r=\{0\} .
$$

These relations imply that $L_{0}^{\infty}(\omega)^{*}$ is a noncommutative Banach algebra. It also follows that $L_{0}^{\infty}(\omega)^{*}$ is not a prime ring. Therefore, we cannot apply the well-known results concerning derivations of commutative Banach algebra and derivation of prime rings to $L_{0}^{\infty}(\omega)^{*}$. It is natural to ask whether the results hold for $L_{0}^{\infty}(\omega)^{*}$. In this paper we investigate the truth of these results for $L_{0}^{\infty}(\omega)^{*}$.

This paper is organized as follows: In Section 2, we prove that the range of every derivation on the noncommutative Banach algebra $L_{0}^{\infty}(\omega)^{*}$ is contained in the radical of $L_{0}^{\infty}(\omega)^{*}$ and that every derivation on $L_{0}^{\infty}(\omega)^{*}$ leaves the primitive ideals of $L_{0}^{\infty}(\omega)^{*}$ invariant. We also show that a derivation on $L_{0}^{\infty}(\omega)^{*}$ is continuous if and only if its restriction to $\operatorname{rad}\left(L_{0}^{\infty}(\omega)^{*}\right)$ is continuous. In Section 3 , we study centralizing derivations and inner derivations of $L_{0}^{\infty}(\omega)^{*}$. We show that there is no nonzero centralizing derivation on $L_{0}^{\infty}(\omega)^{*}$ and prove that the space of all inner derivations of $L_{0}^{\infty}(\omega)^{*}$ is continuously homomorphic to the space $L_{0}^{\infty}(\omega)^{*} / L^{1}(\omega)$.

\section{The range and automatic continuity of derivations}

Let $\operatorname{rad}\left(L_{0}^{\infty}(\omega)^{*}\right)$ denote the radical of Banach algebra $L_{0}^{\infty}(\omega)^{*}$. The main result of this paper is the following theorem.

Theorem 2.1. Let $\omega$ be a weight function on $\mathbb{R}^{+}$and $D$ be a derivation on $L_{0}^{\infty}(\omega)^{*}$. Then $D$ maps $L_{0}^{\infty}(\omega)^{*}$ into $\operatorname{rad}\left(L_{0}^{\infty}(\omega)^{*}\right)=\operatorname{rAnn}\left(L_{0}^{\infty}(\omega)^{*}\right)$.

Proof. First, note that every element of $\operatorname{rAnn}\left(L_{0}^{\infty}(\omega)^{*}\right)$ is nilpotent. So

$$
\operatorname{rAnn}\left(L_{0}^{\infty}(\omega)^{*}\right) \subseteq \operatorname{rad}\left(L_{0}^{\infty}(\omega)^{*}\right) .
$$

Since $\omega(x) \geq 1$ for all $x \in \mathbb{R}^{+}$, the Banach algebra $M(\omega)$ is semisimple; see for example [3]. This together with the fact that $M(\omega)$ is isometrically isomorphism to $L_{0}^{\infty}(\omega)^{*} / \mathrm{r} \operatorname{Ann}\left(L_{0}^{\infty}(\omega)\right)^{*}$ yields that

$$
\operatorname{rad}\left(L_{0}^{\infty}(\omega)^{*}\right) / \operatorname{rAnn}\left(L_{0}^{\infty}(\omega)^{*}\right)=0 .
$$

It follows that $\operatorname{rad}\left(L_{0}^{\infty}(\omega)^{*}\right)$ is contained in $\operatorname{rAnn}\left(L_{0}^{\infty}(\omega)^{*}\right)$. Thus

$$
\operatorname{rad}\left(L_{0}^{\infty}(\omega)^{*}\right)=\operatorname{rAnn}\left(L_{0}^{\infty}(\omega)^{*}\right) .
$$

For every $k \in L_{0}^{\infty}(\omega)^{*}$ and $r \in \operatorname{rAnn}\left(L_{0}^{\infty}(\omega)^{*}\right)$ we have

$$
k \cdot D(r)=D(k \cdot r)-D(k) \cdot r=0 .
$$


Hence $D$ maps $\operatorname{rAnn}\left(L_{0}^{\infty}(\omega)^{*}\right)$ into $\operatorname{rAnn}\left(L_{0}^{\infty}(\omega)^{*}\right)$, which implies that the function

$$
\bar{D}: L_{0}^{\infty}(\omega)^{*} / \operatorname{rAnn}\left(L_{0}^{\infty}(\omega)^{*}\right) \rightarrow L_{0}^{\infty}(\omega)^{*} / \operatorname{rAnn}\left(L_{0}^{\infty}(\omega)^{*}\right)
$$

defined by

$$
\bar{D}\left(m+\operatorname{rAnn}\left(L_{0}^{\infty}(\omega)^{*}\right)\right)=D(m)+\operatorname{rAnn}\left(L_{0}^{\infty}(\omega)^{*}\right)
$$

is well defined. It is easy to see that $\bar{D}$ is a derivation on semisimple and commutative Banach algebra $L_{0}^{\infty}(\omega)^{*} / \operatorname{rAnn}\left(L_{0}^{\infty}(\omega)^{*}\right)$. Thus

$$
\bar{D}\left(m+\operatorname{rAnn}\left(L_{0}^{\infty}(\omega)^{*}\right)\right)=0
$$

for all $m \in L_{0}^{\infty}(\omega)^{*}$. Hence $D(m)$ is an element in $\operatorname{rAnn}\left(L_{0}^{\infty}(\omega)^{*}\right)$. That is,

$$
D\left(L_{0}^{\infty}(\omega)^{*}\right) \subseteq \operatorname{rAnn}\left(L_{0}^{\infty}(\omega)^{*}\right),
$$

as claimed.

As an immediate consequence of this theorem we have the following result.

Corollary 2.2. Let $\omega$ be a weight function on $\mathbb{R}^{+}$. Then the following statements hold:

(i) Any derivation on $L_{0}^{\infty}(\omega)^{*}$ leaves primitive ideals of $L_{0}^{\infty}(\omega)^{*}$ invariant.

(ii) The composition of two derivations on $L_{0}^{\infty}(\omega)^{*}$ is always a derivation on $L_{0}^{\infty}(\omega)^{*}$.

Let us recall that a linear mapping $T: L_{0}^{\infty}(\omega)^{*} \rightarrow L_{0}^{\infty}(\omega)^{*}$ is called spectrally bounded if there exists $\alpha \geq 0$ such that $r(T(m)) \leq \alpha r(m)$ for all $m \in L_{0}^{\infty}(\omega)^{*}$, where $r(\cdot)$ stands for the spectral radius.

Corollary 2.3. Let $\omega$ be a weight function on $\mathbb{R}^{+}$. Then the following statements hold:

(i) Any derivation on $L_{0}^{\infty}(\omega)^{*}$ is spectrally bounded.

(ii) Zero is the only weak*-weak $k^{*}$ continuous derivation on $L_{0}^{\infty}(\omega)^{*}$.

Proof. (i) Let $D$ be a derivation on $L_{0}^{\infty}(\omega)^{*}$. If $m \in L_{0}^{\infty}(\omega)^{*}$, then by Theorem 2.1, $D(m)^{i}=0$ for all $i \geq 2$. Hence

$$
r(D(m))=\lim _{i \rightarrow \infty}\left\|D(m)^{i}\right\|^{1 / i}=0 .
$$

So $D$ is spectrally bounded.

(ii) Let $D$ be a weak*-weak* continuous derivation on $L_{0}^{\infty}(\omega)^{*}$ and $\phi \in$ $L^{1}(\omega)$. Invoke Cohen's factorization theorem to conclude that $\phi=\phi_{1} * \phi_{2}$ for some $\phi_{1}, \phi_{2} \in L^{1}(\omega)$. By definition of $\operatorname{rAnn}\left(L_{0}^{\infty}(\omega)^{*}\right)$, we have

$$
L^{1}(\omega) \cdot \operatorname{rAnn}\left(L_{0}^{\infty}(\omega)^{*}\right)=\{0\} .
$$

Proposition 2.3 of [10] together with the fact that $\operatorname{rAnn}\left(L_{0}^{\infty}(\omega)^{*}\right)=C_{0}(\omega)^{\perp}$ implies that

$$
\operatorname{rAnn}\left(L_{0}^{\infty}(\omega)^{*}\right) \cdot L^{1}(\omega)=\{0\}
$$


From (2) and (3) it follows that

$$
D(\phi)=D\left(\phi_{1} * \phi_{2}\right)=D\left(\phi_{1}\right) \cdot \phi_{2}+\phi_{1} \cdot D\left(\phi_{2}\right)=0 .
$$

This shows that $D\left(L^{1}(\omega)\right)=\{0\}$. From weak* density of $L^{1}(\omega)$ in $L_{0}^{\infty}(\omega)^{*}$ we infer that $D=0$. Hence (ii) holds.

We conclude the section by the following result.

Theorem 2.4. Let $\omega$ be a weight function on $\mathbb{R}^{+}$and $D$ be a derivation on $L_{0}^{\infty}(\omega)^{*}$. Then $D$ is continuous if and only if $\left.D\right|_{\operatorname{rad}\left(L_{0}^{\infty}(\omega)^{*}\right)}$ is continuous.

Proof. Choose $u \in \Lambda\left(L_{0}^{\infty}(\omega)^{*}\right)$. Let $\left(u \cdot m_{\alpha}\right)_{\alpha \in A}$ be a net in $L_{0}^{\infty}(\omega)^{*}$ such that

$$
u \cdot m_{\alpha} \rightarrow 0 \quad \text { and } \quad D\left(u \cdot m_{\alpha}\right) \rightarrow m \in L_{0}^{\infty}(\omega)^{*}
$$

in the norm topology of $L_{0}^{\infty}(\omega)^{*}$. Suppose that $m \neq 0$ and $i \in \mathbb{N}$. Choose $\alpha_{0} \in A$ such that $\left\|D\left(u \cdot m_{\alpha}\right)\right\| \geq\|m\| / 2$ and $\left\|u \cdot m_{\alpha}\right\|<\|m\| / i$ for all $\alpha \geq \alpha_{0}$. In view of Theorem 2.1, we have

$$
\begin{aligned}
\|m\| / 2 & \leq\left\|D\left(u \cdot m_{\alpha}\right)\right\|=\left\|D(u) \cdot m_{\alpha}\right\| \\
& =\left\|D(u) \cdot u \cdot m_{\alpha}\right\| \leq\|D(u)\|\left\|u \cdot m_{\alpha}\right\| \\
& \leq\|D(u)\|\|m\| / i .
\end{aligned}
$$

Hence $\|D(u)\| \geq i / 2$ for all $i \in \mathbb{N}$. This contradiction shows that $m=0$. By closed graph theorem, $\left.D\right|_{u \cdot L_{0}^{\infty}(\omega) *}$ is continuous. Thus there exists $M_{1}>0$ such that for every $m \in L_{0}^{\infty}(\omega)^{*}$

$$
\|D(u \cdot m)\| \leq M_{1}\|u \cdot m\| .
$$

Now, let $\left.D\right|_{\operatorname{rad}\left(L_{0}^{\infty}(\omega)^{*}\right)}$ be continuous. Then there exists $M_{2}>0$ such that $\|D(r)\| \leq M_{2}\|r\|$ for all $r \in \operatorname{rad}\left(L_{0}^{\infty}(\omega)^{*}\right)$. For any $m \in L_{0}^{\infty}(\omega)^{*}$ set

$$
r_{m}=m-u \cdot m \in \operatorname{rad}\left(L_{0}^{\infty}(\omega)^{*}\right) \text {. }
$$

Then

$$
\begin{aligned}
\|D(m)\| & =\left\|D(u \cdot m)+D\left(r_{m}\right)\right\| \\
& \leq\|D(u \cdot m)\|+\left\|D\left(r_{m}\right)\right\| \\
& \leq M_{1}\|u \cdot m\|+M_{2}\|m-u \cdot m\| \\
& \leq M_{1}\|m\|+2 M_{2}\|m\| \\
& =\left(M_{1}+2 M_{2}\right)\|m\| .
\end{aligned}
$$

Therefore, $D$ is bounded. The converse is clear.

\section{Centralizing derivations and inner derivations}

In the sequel, $\mathrm{Z}\left(L_{0}^{\infty}(\omega)^{*}\right)$ denotes the center of $L_{0}^{\infty}(\omega)^{*}$ and we write $[m, n]=$ $m \cdot n-n \cdot m$ for all $m, n \in L_{0}^{\infty}(\omega)^{*}$.

Theorem 3.1. Let $\omega$ be a weight function on $\mathbb{R}^{+}$and $D$ be a derivation on $L_{0}^{\infty}(\omega)^{*}$. Then the following assertions are equivalent. 
(a) $D=0$.

(b) There exists $i \in \mathbb{N}$ such that $D\left(m^{i}\right)=0$ for all $m \in L_{0}^{\infty}(\omega)^{*}$.

(c) There exists $i \in \mathbb{N}$ such that $D\left(m^{i}\right) \in Z\left(L_{0}^{\infty}(\omega)^{*}\right)$ for all $m \in L_{0}^{\infty}(\omega)^{*}$.

(d) There exists $i \in \mathbb{N}$ such that $\left[D(m), m^{i}\right] \in Z\left(L_{0}^{\infty}(\omega)^{*}\right)$ for all $m \in$ $L_{0}^{\infty}(\omega)^{*}$.

(e) There exists $i \in \mathbb{N}$ such that $\left[D(m), m^{i}\right]=0$ for all $m \in L_{0}^{\infty}(\omega)^{*}$.

Proof. The implications $(\mathrm{a}) \Rightarrow(\mathrm{b}) \Rightarrow(\mathrm{c})$ are clear. It follows from Theorem 2.1 that

$$
\left[D(m), m^{i-1}\right]=D(m) \cdot m^{i-1}-m^{i-1} \cdot D(m)=D\left(m^{i}\right) .
$$

Hence $(\mathrm{c}) \Rightarrow(\mathrm{d})$. Let us show that $(\mathrm{d}) \Rightarrow(\mathrm{e})$ and $(\mathrm{e}) \Rightarrow(\mathrm{a})$. Assume that $(\mathrm{d})$ holds. Choose $u \in \Lambda\left(L_{0}^{\infty}(\omega)^{*}\right)$. Then

$$
\begin{aligned}
{\left[D(m), m^{i}\right] } & =\left[D(m), m^{i}\right] \cdot u=u \cdot\left[D(m), m^{i}\right] \\
& =u \cdot D(m) \cdot m^{i}=0 .
\end{aligned}
$$

So, we obtain (e). To complete the proof, suppose that there is $i \in \mathbb{N}$ such that $\left[D(m), m^{i}\right]=0$ for all $m \in L_{0}^{\infty}(\omega)^{*}$. Fix $u \in \Lambda\left(L_{0}^{\infty}(\omega)^{*}\right)$. Then

$$
D(u)=\left[D(u), u^{i}\right]=0 .
$$

For every $r \in \operatorname{rAnn}\left(L_{0}^{\infty}(\omega)^{*}\right)$, we have

$$
\begin{aligned}
0 & =\left[D(r+u),(r+u)^{i}\right]=\left[D(r),(r+u)^{i}\right] \\
& =D(r)\left(r^{i}+c_{1} r^{i-1} u+c_{2} r^{i-2} u^{2}+\cdots+c_{i-1} r u^{i-1}+u^{i}\right)=D(r)
\end{aligned}
$$

for some $c_{1}, \ldots, c_{i-1} \geq 0$. Now, let $m \in L_{0}^{\infty}(\omega)^{*}$. Then

$$
r_{m}=m-u \cdot m \in \operatorname{rAnn}\left(L_{0}^{\infty}(\omega)^{*}\right) .
$$

Thus

$$
D(m)=D(u \cdot m)+D\left(r_{m}\right)=D(u) \cdot m+D\left(r_{m}\right)=0 .
$$

Therefore, $D=0$. So (e) implies (a).

A mapping $T: L_{0}^{\infty}(\omega)^{*} \rightarrow L_{0}^{\infty}(\omega)^{*}$ is called centralizing if $[T(m), m] \in$ $\mathrm{Z}\left(L_{0}^{\infty}(\omega)^{*}\right)$ for all $m \in L_{0}^{\infty}(\omega)^{*}$; in a special case when $[T(m), m]=0$ for all $m \in L_{0}^{\infty}(\omega)^{*}, T$ is called commuting.

Corollary 3.2. Let $\omega$ be a weight function on $\mathbb{R}^{+}$. Then the following statements hold:

(i) Zero is the only centralizing derivation on $L_{0}^{\infty}(\omega)^{*}$.

(ii) Zero is the only commuting derivation on $L_{0}^{\infty}(\omega)^{*}$.

A derivation $D$ on $L_{0}^{\infty}(\omega)^{*}$ is said to be inner if there exists $n_{0} \in L_{0}^{\infty}(\omega)^{*}$ such that $D(m)=\left[m, n_{0}\right]$ for all $m \in L_{0}^{\infty}(\omega)^{*}$.

Proposition 3.3. Let $\omega$ be a weight function on $\mathbb{R}^{+}$and $D$ be a derivation on $L_{0}^{\infty}(\omega)^{*}$. Then the following assertions are equivalent.

(a) $D$ is inner. 
(b) There exists $n_{0} \in L_{0}^{\infty}(\omega)^{*}$ such that $D\left(m^{2}\right)=\left[m^{2}, n_{0}\right]$ for all $m \in$ $L_{0}^{\infty}(\omega)^{*}$.

(c) There exists $n_{0} \in L_{0}^{\infty}(\omega)^{*}$ such that the mapping $m \mapsto D(m)+n_{0} \cdot m$ is commuting.

(d) There exists $n_{0} \in L_{0}^{\infty}(\omega)^{*}$ such that the mapping $m \mapsto D(m)+n_{0} \cdot m$ is centralizing.

Proof. It is obvious that (a) implies (b) and that (c) implies (d). Suppose that there is $n_{0} \in L_{0}^{\infty}(\omega)^{*}$ such that $D\left(m^{2}\right)=\left[m^{2}, n_{0}\right]$ for all $m \in L_{0}^{\infty}(\omega)^{*}$, then by (1) we have

$$
\begin{aligned}
{\left[D(m)+n_{0} \cdot m, m\right] } & =D(m) \cdot m+n_{0} \cdot m^{2}-m \cdot n_{0} \cdot m \\
& =D\left(m^{2}\right)+n_{0} \cdot m^{2}-m \cdot n_{0} \cdot m \\
& =\left(m^{2} \cdot n_{0}-n_{0} \cdot m^{2}\right)+n_{0} \cdot m^{2}-m^{2} \cdot n_{0} \\
& =0 .
\end{aligned}
$$

This shows that the mapping $m \mapsto D(m)+n_{0} \cdot m$ is commuting. So (b) implies (c). Finally, let $n_{0} \in L_{0}^{\infty}(\omega)^{*}$. We define $\tilde{D}: L_{0}^{\infty}(\omega)^{*} \rightarrow L_{0}^{\infty}(\omega)^{*}$ by

$$
\tilde{D}(m)=D(m)-\left[m, n_{0}\right] .
$$

It is plain that $\tilde{D}$ is a derivation on $L_{0}^{\infty}(\omega)^{*}$. It follows from Theorem 2.1 that

$$
[\tilde{D}(m), m]=\tilde{D}(m) \cdot m=\left[D(m)+n_{0} \cdot m, m\right] .
$$

If (d) holds, then $\tilde{D}$ is a centralizing derivation. So $\tilde{D}(m)=0$ by Corollary 3.2. This shows that

$$
D(m)=\left[m, n_{0}\right]
$$

for all $m \in L_{0}^{\infty}(\omega)^{*}$. That is, $D$ is inner. Hence (d) implies (a).

In the following, let $\operatorname{InnD}\left(L_{0}^{\infty}(\omega)^{*}\right)$ be the space of all inner derivations on $L_{0}^{\infty}(\omega)^{*}$.

Theorem 3.4. Let $\omega$ be a weight function on $\mathbb{R}^{+}$. Then the mapping $\mathcal{I}$ : $m+L^{1}(\omega) \mapsto \mathcal{I}_{m}$ is a continuous homomorphism from $L_{0}^{\infty}(\omega)^{*} / L^{1}(\omega)$ onto Inn $\mathrm{D}\left(L_{0}^{\infty}(\omega)^{*}\right)$, where $\mathcal{I}_{m}(n)=[n, m]$ for all $m \in L_{0}^{\infty}(\omega)^{*}$. Furthermore, $\mathcal{I}$ is an isomorphism if and only if $Z\left(L_{0}^{\infty}(\omega)^{*}\right)=L^{1}(\omega)$.

Proof. First note that if $u \in \Lambda\left(L_{0}^{\infty}(\omega)^{*}\right)$, then $u$ is a mixed identity and $u$. $L_{0}^{\infty}(\omega)^{*}$ is commutative. Let $m \in L_{0}^{\infty}(\omega)^{*}$ and $\phi \in L^{1}(\omega)$. Then

$$
\begin{aligned}
\phi \cdot m & =u \cdot \phi \cdot u \cdot m=u \cdot m \cdot u \cdot \phi \\
& =u \cdot m \cdot \phi .
\end{aligned}
$$

Since $L^{1}(\omega)$ is an ideal in $L_{0}^{\infty}(\omega)^{*}$ and $u$ is a mixed identity, we get

$$
u \cdot m \cdot \phi=m \cdot \phi \text {. }
$$

From (4) and (5) we see that

$$
\phi \cdot m=m \cdot \phi .
$$


So the mapping $\mathcal{I}$ is well defined. Obviously, $\mathcal{I}$ is a homomorphism. To see that $\mathcal{I}$ is continuous, let $n \in L_{0}^{\infty}(\omega)^{*}$ and $\phi \in L^{1}(\omega)$. Then

$$
\begin{aligned}
\left\|\mathcal{I}_{m}(n)\right\| & =\|n \cdot m-m \cdot n\| \\
& \leq\|n \cdot m-\phi \cdot n\|+\|\phi \cdot n-m \cdot n\| \\
& \leq\|n\|\|m-\phi\|+\|\phi-m\|\|n\| \\
& =2\|n\|\|m-\phi\|
\end{aligned}
$$

for all $m \in L_{0}^{\infty}(\omega)^{*}$. This implies that

$$
\left\|\mathcal{I}\left(m+L^{1}(\omega)\right)\right\|=\left\|\mathcal{I}_{m}\right\| \leq 2\|m-\phi\|
$$

for all $m \in L_{0}^{\infty}(\omega)^{*}$ and $\phi \in L^{1}(\omega)$. Hence

$$
\begin{aligned}
\left\|\mathcal{I}\left(m+L^{1}(\omega)\right)\right\| & \leq 2 \inf \left\{\|m-\phi\|: \phi \in L^{1}(\omega)\right\} \\
& =2 \inf \left\{\|m+\phi\|: \phi \in L^{1}(\omega)\right\}=2\left\|m+L^{1}(\omega)\right\| .
\end{aligned}
$$

Therefore, $\mathcal{I}$ is continuous.

Now, let $m \in \mathrm{Z}\left(L_{0}^{\infty}(\omega)^{*}\right)$. Then $\mathcal{I}_{m}=0$ on $L_{0}^{\infty}(\omega)^{*}$. Thus $\mathcal{I}\left(m+L^{1}(\omega)\right)=0$. It follows that $m \in L^{1}(\omega)$ if $\mathcal{I}$ is an isomorphism. This together with (6) shows that

$$
\mathrm{Z}\left(L_{0}^{\infty}(\omega)^{*}\right)=L^{1}(\omega) .
$$

To complete the proof, let $m \in L_{0}^{\infty}(\omega)^{*}$ and $\mathcal{I}\left(m+L^{1}(\omega)\right)=0$. Then

$$
\mathcal{I}_{m}(n)=n \cdot m-m \cdot n=0
$$

for all $n \in L_{0}^{\infty}(\omega)^{*}$. This shows that $m \in \mathrm{Z}\left(L_{0}^{\infty}(\omega)^{*}\right)$. So, if $\mathrm{Z}\left(L_{0}^{\infty}(\omega)^{*}\right)=$ $L^{1}(\omega)$, then $m \in L^{1}(\omega)$ and so $\mathcal{I}$ is an isomorphism.

Acknowledgement. The authors would like to thank the referee of the paper for invaluable comments.

\section{References}

[1] M. Bresar and M. Mathieu, Derivations mapping into the radical III, J. Funct. Anal. 133 (1995), no. 1, 21-29.

[2] J. W. Conway, A Course in Functional Analysis, Springer-Verlag, New York, 1985.

[3] H. G. Dales, Banach Algebras and Automatic Continuity, Oxford University Press, New York, 2000.

[4] N. Isik, J. Pym, and A. Ülger, The second dual of the group algebra of a compact group, J. London Math. Soc. 35 (1987), no. 1, 135-148.

[5] B. E. Johnson, Continuity of derivations on commutative algebras, Amer. J. Math. 91 (1969), 1-10.

[6] K. W. Jun and H. M. Kim, Derivations on prime rings and Banach algebras, Bull. Korean Math. Soc. 38 (2001), no. 4, 709-718.

[7] _ Approximate derivations mapping into the radicals of Banach algebras, Taiwanese J. Math. 11 (2007), no. 1, 277-288.

[8] A. T. Lau and J. Pym, Concerning the second dual of the group algebra of a locally compact group, J. London Math. Soc. 41 (1990), no. 3, 445-460. 
[9] S. Maghsoudi, M. J. Mehdipour, and R. Nasr-Isfahani, Compact right multipliers on a Banach algebra related to locally compact semigroups, Semigroup Forum 83 (2011), no. 2, 205-213.

[10] S. Maghsoudi, R. Nasr-Isfahani, and A. Rejali, Arens multiplication on Banach algebras related to locally compact semigroups, Math. Nachr. 281 (2008), no. 10, 1495-1510.

[11] M. Mathieu and G. J. Murphy, Derivations mapping into the radical, Arch. Math. 57 (1991), no. 5, 469-474.

[12] M. Mathieu and V. Runde, Derivations mapping into the radical II, Bull. London Math. Soc. 24 (1992), no. 5, 485-487.

[13] A. R. Medghalchi, The second dual algebra of a hypergroup, Math. Z. 210 (1992), no. $4,615-624$.

[14] S. Ouzomgi, Factorization and bounded approximate identities for a class of convolution Banach algebras, Glasg. Math. J. 28 (1986), no. 2, 211-214.

[15] E. C. Posner, Derivations in prime rings, Proc. Amer. Math. Soc. 8 (1957), 1093-1100.

[16] A. M. Sinclair, Continuous derivations on Banach algebras, Proc. Amer. Math. Soc. 20 (1969), no. 1, 166-170

[17] I. M. Singer and J. Wermer, Derivations on commutative normed algebras, Math. Ann. 129 (1955), 260-264.

[18] A. I. Singh, $L_{0}^{\infty}(G)^{*}$ as the second dual of the group algebra $L^{1}(G)$ with a locally convex toplogy, Michigan Math. J. 46 (1999), no. 1, 143-150.

[19] M. Thomas, The image of a derivation is contained in the radical, Ann. of Math. 128 (1988), no. 3, 435-460

[20] J. Vukman, On left Jordan derivations of rings and Banach algebras, Aequationes Math. 75 (2008), no. 3, 260-266.

Mohammad Javad Mehdipour Department of Mathematics Shiraz University of TeChNology SHIRAZ 71555-313, IRAN

E-mail address: mehdipour@sutech.ac.ir

Zahra SAeEdi

Department of Mathematics

Shiraz University of TeChNology

SHIRAZ 71555-313, IRAN

E-mail address: asetarehsorkh@yahoo.com 